e-migrinter

e-Migrinter

$14 \mid 2016$

L'ajustement méthodologique comme fabrique critique du savoir dans les études migratoires

\title{
Une invitation aux enquêtes transnationales
}

Retour sur le projet TIMME (Terrains Interdisciplinaires et Multi-sites : Migrations et Engagements)

\section{Ilka Vari-Lavoisier}

\section{(2) OpenEdition}

\section{Journals}

Édition électronique

URL : https://journals.openedition.org/e-migrinter/712

DOI : 10.4000/e-migrinter.712

ISSN : 1961-9685

\section{Éditeur}

UMR 7301 - Migrinter

\section{Référence électronique}

Ilka Vari-Lavoisier, "Une invitation aux enquêtes transnationales », e-Migrinter [En ligne], 14 | 2016, mis en ligne le, consulté le 20 mai 2021. URL : http://journals.openedition.org/e-migrinter/712 ; DOI :

https://doi.org/10.4000/e-migrinter.712

Ce document a été généré automatiquement le 20 mai 2021.

Tous droits réservés 


\title{
Une invitation aux enquêtes transnationales
}

\author{
Retour sur le projet TIMME (Terrains Interdisciplinaires et Multi-sites : \\ Migrations et Engagements)
}

\section{Ilka Vari-Lavoisier}

\section{NOTE DE L'AUTEUR}

Ce projet a été financé par une subvention octroyée au laboratoire DIAL par la Mairie de Paris, dans le cadre des appels à projets « Emergence(s)». Les auteurs tiennent à remercier les coordinatrices du projet et plus largement l'équipe de DIAL pour avoir rendu ce projet possible, tant par leur appui financier, logistique et scientifique.

Avant-propos

Capter la complexité d'un monde en mouvement est un défi méthodologique passionnant, et le projet TIMME s'inscrit dans l'effort de forger des outils empiriques adaptés à l'analyse des flux qui parcourent nos sociétés. Aussi, loin de chercher à

résumer les conclusions de l'enquête TIMME

(nous revoyons pour cela aux travaux de Dompierre-Major, 2012 ; Grysole, 2012; Vari-

Lavoisier, 2015), ce texte décrit la mise en pratique de stratégies concrètes visant à surmonter les difficultés méthodologiques rencontrées dans le contexte des études migratoires. En donnant à voir les forces et les

limites de ce projet, ce texte espère inciter d'autres chercheurs à se lancer dans des aventures collectives transnationales. 
Cette enquête est en effet d'abord née d'une collaboration entre Flore Gubert, Sandrine Mesplé-Somps et Lisa Chauvet, économistes du

laboratoire Institut de Recherche pour le

Développement (DIAL). L'équipe a ensuite accueilli des politistes et sociologues. Malgré les limites des lignes qui vont suivre, ce retour d'expérience essaie de rendre humblement hommage à leur travail.

\section{Introduction}

1 Notre $^{1}$ collectif de recherche s'est formé autour d'un intérêt commun pour les conséquences des migrations internationales, et plus précisément sur les transferts d'argent des migrants. Le cas des ressortissants sénégalais, vivant en France semblait d'autant plus intéressant que leurs transferts ont contribué à la formalisation d'un tissu d'associations de migrants qui portent des projets de développement, entre la France et le Sénégal. Or, pour étudier le fonctionnement de ces associations de ressortissants ${ }^{2}$, il était impossible de confiner notre enquête à un lieu unique (France ou Sénégal) puisque la structuration de ces associations épouse les routes migratoires avec généralement trois antennes (Dia, 2008; Lacroix et al., 2008) : une dans la localité d'installation des migrants (très largement en île-de-France) ${ }^{3}$, une antenne dans leur village natal au Sénégal, et un relais dans la capitale de départ (Dakar). Étudier ces entités séparément reviendrait à fragmenter l'espace transnational où elles évoluent, sans pouvoir étudier la consistance de leurs relations "systémiques » (Lacroix, 2003 : 44). Afin d'analyser ces structures dans toute leur complexité, nous avons misé sur l'intelligence collective et conçu un protocole d'enquête transnational, interdisciplinaire et collaboratif: le dispositif «Terrains Interdisciplinaires et Multi-sites sur les Migrants et leurs Engagements » (TIMME).

\section{Genèse du projet (phase 1, cf. poster)}


Figure $n^{\circ} 1$ : Poster scientifique - TIMME

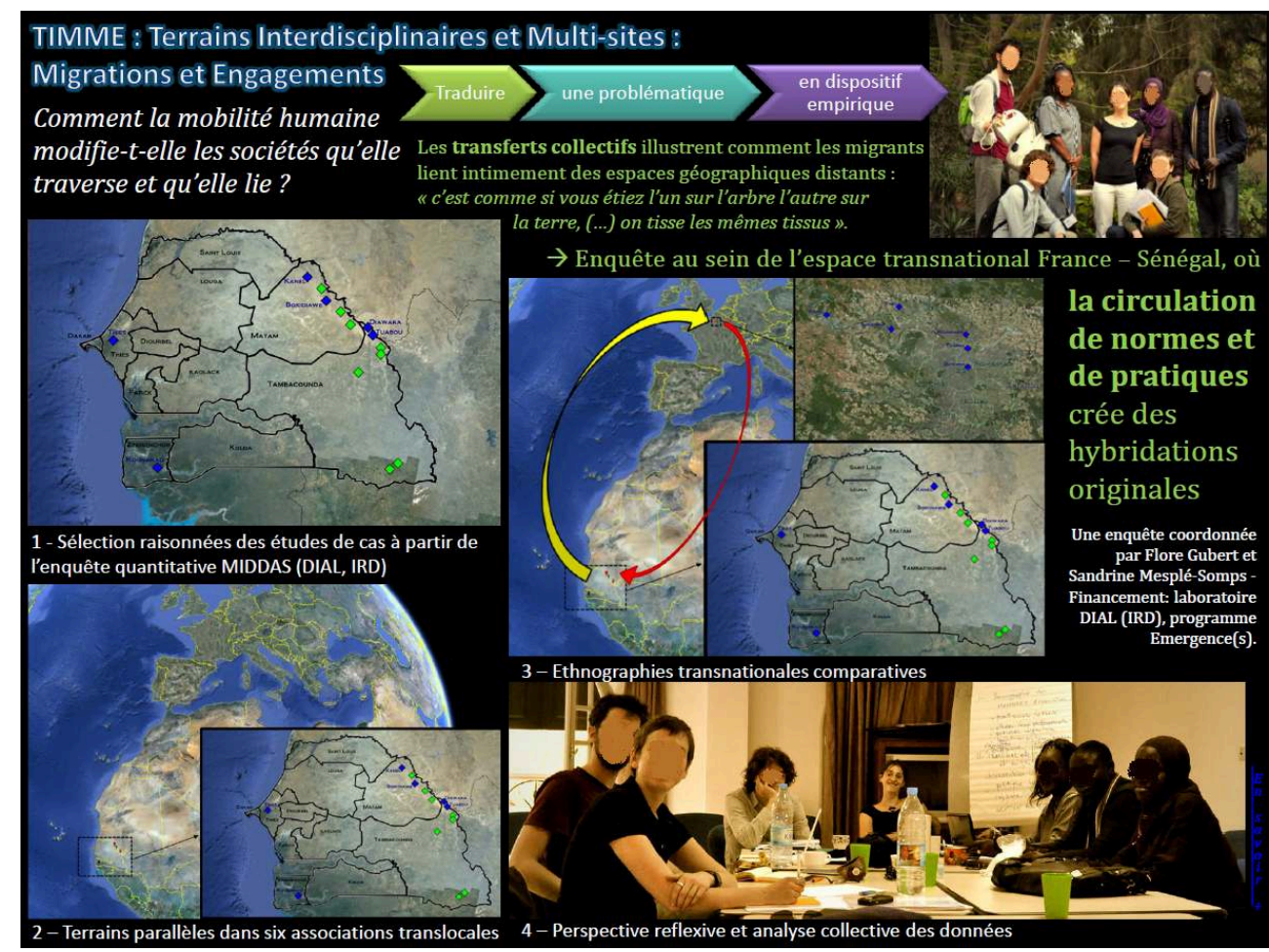

Source : Sané et Vari-Lavoisier, 2014.

2 L'enquête TIMME s'inscrit dans le prolongement du projet « Migrations Internationales et Développement: une analyse à partir de Données Appariées migrants-familles d'origine au Sénégal »(MIDDAS), un projet conçu et mené au sein du laboratoire Développement Institution et Mondialisation (DIAL) ${ }^{4}$.

3 L'enquête MIDDAS (2006 - 2009) visait à mieux estimer les canaux et les usages des transferts des migrants. Élaboré par Flore Gubert, avec l'appui de Sandrine MespléSomps et de Lisa Chauvet (économistes du laboratoire DIAL) et en collaboration avec Claire Bernard, le questionnaire interrogeait des ressortissants sénégalais sur leurs parcours migratoires, leur situation socio-économique avant et pendant la migration, leurs réseaux d'interconnaissances et leurs transferts économiques.

4 L'analyse des données appariées a révélé que les réseaux d'interconnaissances des migrants (composé le plus souvent de parents éloignés et de ressortissants issus du même village ou de la même ethnie), jouent un rôle décisif dans le maintien du système d'obligations qui incite les migrants à redistribuer les bénéfices migratoires. Cela éclaire la proportion importante de migrants qui envoient des fonds au Sénégal : plus de $41 \%$ des émigrés sont concernés, quel que soit leur pays de destination. Parmi les migrants membres d'associations, cette proportion atteint $60 \%$ (TeO, INED, 2008). Ces données ont suscité l'envie de mieux comprendre le rôle et le fonctionnement des associations de migrants.

5 Trois cent questionnaires ont été complétés en France et trois cent en Italie (au sein d'échantillons stratifiés par zones géographiques pour être représentatifs à l'échelle nationale). Malgré les difficultés logistiques, pour retrouver les ménages d'origine difficultés inhérentes aux enquêtes quantitatives transnationales (Beauchemin et 
González-Ferrer, 2010) -, près d'un tiers des familles a répondu à l'enquête « miroir » au Sénégal (31,7 \%).

6 L'enquête MIDDAS a ainsi le mérite de ne pas utiliser l'effet "boule de neige ", technique d'échantillonnage dont les biais sont les plus lourds (McKenzie et Mistiaen, 2009:356) si l'on cherche à estimer la fréquence d'une pratique, car la progression au sein d'un réseau d'interconnaissances tend à favoriser les contacts avec des enquêtés aux pratiques homogènes. Le dispositif MIDDAS permettait donc d'enquêter aussi des migrants ayant rompu tout contact avec leur ménage d'origine, ou encore des migrants en situation irrégulière. Cette enquête repose ainsi sur une méthode d'échantillonnage qui permet de mieux évaluer la fréquence des pratiques transnationales, dont l'associationnisme (Beauchemin et González-Ferrer, 2010, 16-20).

\section{Du multi-site au transnational}

7 Contrairement aux enquêtes quantitatives transnationales (qui se multiplient ces dernières années (Beauchemin et al., 2014), les exemples d'enquêtes qualitatives transnationales restent marginaux. Malgré les appels en ce sens (Burawoy, 2003; Marcus, 1995 ; Mazzucato et Schans, 2011), leur mise en œuvre reste rare, d'abord pour des raisons matérielles (notamment financières) évidentes (Ahmed, 2009). Les premières enquêtes qualitatives transnationales émanent de l'Université de Princeton (États-Unis). L'initiative la plus connue, le «Mexican Migration Project », a été initiée en 1982, par Jorge Durand (Guadalajara, Mexique) et Douglas S. Massey (Princeton, États-Unis) pour mieux comprendre les conditions de vie des migrants mexicains vivant aux États-Unis. Mais à l'exception de cette enquête, les collectes de données qualitatives sur les migrations sont généralement menées soit sur le lieu d'installation des migrants ${ }^{5}$, soit dans le pays d'origine des migrants ${ }^{6}$ et ne permettent pas d'enquêter cet «espace de flux » entre pays d'origine et de destination, qui nous intéressait justement.

8 Notre terrain n'a donc pas été tant délimité par des contingences matérielles que par l'objet de recherche (les transferts) qui invitait à retracer les trajectoires et allersvenues des migrants, depuis les zones rurales du Sénégal aux capitales, de Dakar à Paris. Cette approche a permis de saisir la pluralité des stratégies mises en œuvre par des acteurs aux pratiques, discours et référentiels dispersés géographiquement, mais fortement liés socialement (Buscher, Urry, 2009).

9 Rendre compte de la mobilité des personnes et suivre leurs déplacements (Marcus, 1995) est un préalable pour constituer la mobilité géographique en champ d'investigation empirique (Faist, 2012). Le dispositif d'enquête devait donc à la fois restituer la mobilité de certains acteurs (et la façon dont cette mobilité a une incidence sur leurs stratégies, leurs ressources ...) - et tenir compte du rôle occupé par les absents (ou les présents par intermittence) - dans les différents lieux géographiques liés par ces activités. Ce qui n'est pas sans placer l'ethnographe devant un dilemme. Quelle stratégie privilégier : suivre les migrants pour rendre compte de leurs stratégies? Ou observer, en un lieu, les fluctuations induites par leur présence/absence? Contraintes aussi prosaïques que réelles: sans don d'ubiquité, les bénéfices supposés d'une approche multi-site restent virtuels! En particulier lorsqu'il s'agit d'ethnographie, car construire des relations de long terme avec ses enquêtés est délicat lorsque l'on enquête sur plusieurs sites à la fois (Mazzucato, 2009). Pour ces raisons, la mise en 
œuvre de projets collaboratifs permet de répondre à nombre de défis soulevés par la réalisation d'ethnographies multi-sites (Fitzgerald, 2006:6). Nous avons donc choisi d'élargir l'équipe d'ethnographes, afin de mutualiser le recueil et l'analyse de données qualitatives.

\section{L'union fait la force (du mobile au multi-site)}

10 Pour étudier les transferts transnationaux des migrants, nous voulions suivre les trajectoires des migrants, depuis les zones rurales du Sénégal jusqu'aux capitales, de Dakar à Paris. En d'autres termes, il s'agissait de constituer la mobilité géographique en champ d'investigation empirique. Pour ce faire, deux équipes de chercheurs ont été constituées, l'une à Paris, l'autre à Dakar. Cela permettait de mener l'enquête ethnographique parallèlement, en France et au Sénégal, au sein des six associations de migrants ( $c f$. poster, phase 2).

11 À l'issue de leurs premiers mois de terrain, les ethnographes (français et sénégalais) ont restitué le déroulement de leurs enquêtes, et ont été invités à réfléchir aux similitudes et aux spécificités des cas étudiés (cf. poster, phase 3).

12 Enfin, l'objectif était de mener à bien la dimension transnationale de l'enquête en réunissant toute l'équipe. Quatre ethnographes français se sont donc rendus au Sénégal. Les ethnographes se sont regroupés en binômes binationaux qui avaient travaillé sur la même structure, de part et d'autre du Sahara, et sont partis ensemble sur leurs terrains respectifs pour dix à douze jours d'enquête. À leur retour, les séances collectives de restitution et de réflexion ont été particulièrement riches ( $c f$. poster, phase 4), notamment en offrant un cadre permettant de réfléchir collectivement aux pistes à privilégier pour analyser nos différentes études de cas.

\section{La dream TIMME}

13 Le dispositif TIMME est ainsi un modeste émule de la procédure «Enquête Collective Rapide d'Identification des conflits et des groupes Stratégiques" (ECRIS), mise en œuvre au laboratoire d'Études et de Recherche sur les Dynamiques Sociales et le Développement Local (LASDEL), basée à Niamey (Niger) autour de Jean-Pierre Olivier de Sardan (Bierschenk, de Sardan, 1994). À ce titre, notre expérience confirme, à son échelle, comment la dimension collective invite chaque participant à questionner les choix et les orientations qu'il/elle aurait donné à son enquête s'il (ou elle) l'avait réalisé $\operatorname{seul}(\mathrm{e})$.

14 Plus encore, le groupe auquel nous tendions à nous identifier - et, peut-être plus encore, à être assigné par nos enquêtés -, était d'abord celui de notre couleur de peau et de notre nationalité. Sans pouvoir surmonter ces difficultés, cette enquête a permis de confronter les expériences de différents chercheurs sur un même terrain, posant autant de jalons pour analyser comment nos catégories d'observation, de description, et d'analyse sont façonnées par notre propre parcours et notre identité. 


\section{BIBLIOGRAPHIE}

(2008) Trajectoire et origines (TeO) : enquête sur la diversité des populations en France, Paris, Ined ; Insee.

Ahmed, Yasmine (2009) Multi-sited Ethnography : Potentials and Challenges, IMI Working Paper, $n^{\circ} 11,19 \mathrm{p}$

Beauchemin, Cris ; González-Ferrer, Amparo (2010) Multi-Country Surveys on International Migration : An Assessment of Selection Biases in Destination Countries, MAFE Working Paper, $\mathrm{n}^{\circ} 3$, $43 \mathrm{p}$.

Beauchemin, Cris ; Papa, Sakho ; Schoumaker, Bruno ; Flahaux, Marie-Laurence (2014) New patterns of migration between Senegal and Europe, MAFE Working Paper, $n^{\circ} 21,33$ p. [Disponible sur internett].

Bierschenk, Thomas ; Olivier de Sardan, Jean-Pierre (1994) ECRIS : Enquête Collective Rapide d'Identification des conflits et des groupes Stratégiques..., Bulletin de l'APAD, $\mathrm{n}^{\circ}$ 7, $9 \mathrm{p}$.

Burawoy, Michael (2003) Revisits : an outline of a theory of reflexive ethnography, American Sociological Review, $\mathrm{n}^{\circ} 68$, pp. 645-679.

Büscher, Monika ; Urry, John (2009) Mobile methods and the empirical. European, Journal of Social Theory, $\mathrm{n}^{\circ} 12$, pp. 99-116.

Dia, Hamidou (2008) Villages multi-situés du Fouta-Toro en France : le défi de la transition entre générations de caissiers, lettrés et citadins, Revue Asylon(s), $n^{\circ} 3$ [Disponible sur Internet].

Dompierre-Major, Laurence (2012) Les migrants au sein de la coopération décentralisée. Construction d'un nouvel espace transnational. Le cas des associations Solidarité Cergy Thies et Solidarité Thies Cergy, Paris, Université Paris I Panthéon Sorbonne, 128 p.

Mém. Mast. 2 : Sc. Po. : Paris : 2012.

Faist, Thomas (2012) Toward a Transnational Methodology : Methods to Address Methodological Nationalism, Essentialism, and Positionality, Revue européenne des migrations internationales, vol. $28, n^{\circ} 1$, pp. 51-70.

Fitzgerald, David (2006) Toward a theoretical ethnography of migration, Qualitative Sociology, vol. $29, \mathrm{n}^{\circ} 1$, pp. 1-24.

Grysole, Amélie (2012) Le militantisme économique au service de l'intérêt du village ? Une lutte pour l'insertion socio-politique au sein du village multi-situé de $D^{* * *}$, Paris, École normale supérieure / École des hautes études en sciences sociales, $189 \mathrm{p}$.

Mém. Master 2 :PDI : Paris : 2012.

Lacroix, Thomas (2003) Espace transnational et territoires. Les réseaux marocains du développement, Poitiers, Université de Poitiers, $455 \mathrm{p}$.

Th. Doct : Géogr. : Poitiers : 2003.

Lacroix, Thomas ; Sall, Lela ; Salzbrunn, Monika (2008) Marocains et Sénégalais de France : permanences et évolution des relations transnationales, Revue européenne des migrations internationales, vol. $24, \mathrm{n}^{\circ} 2$, pp. 23-43.

Marcus, George E. (1995) Ethnography in/of the World System : The Emergence of Multi-Sited Ethnography, Annual Review of Anthropology, n²4, pp. 95-117. 
Mazzucato, Valentina (2009) Bridging boundaries with a transnational research approach : A simultaneous matched sample methodology, in Falzon, M.-A. (Ed) Multi-sited ethnography: Theory, praxis and locality in contemporary social research, Hampshire, Ashgate, pp. 215-232.

Mazzucato, Valentina ; Schans, Djamila (2011) Transnational Families and the Well-Being of Children : Conceptual and Methodological Challenges, Journal of Marriage and Family, vol. 73, $\mathrm{n}^{\circ} 4$, pp. 704-712.

McKenzie, David ; Mistiaen, Johan (2009) Surveying migrant households : a comparison of censusbased, snowball and intercept point surveys, Journal of the Royal Statistical Society : Series A

(Statistics in Society), vol. 172, $\mathrm{n}^{\circ} 2$, pp. 339-360.

Vari-Lavoisier, Ilka (2015) La circulation des significations sociales de l'argent. Transferts économiques, sociaux et politiques entre le Sénégal et la France, Paris, École Normale Supérieure de Paris, 467 p., Th. Doct : Sociologie : Paris : 2015.

\section{NOTES}

1. L'usage du pronom «nous » désigne le collectif introduit en avant-propos.

2. Ou "Home Town Associations" dans la littérature anglophone: associations dont le critère d'adhésion est le partage d'une origine commune. Ici il s'agit d'associations françaises de loi 1901 créées par et pour des migrants sénégalais, généralement natifs d'un même village.

3. Comme le confirment les résultats de l'enquête «Migrations internationales et Développement: une analyse des Données appariées migrants-famille d'origine sénégalaise » (MIDDAS) (cf. infra) et le Journal officiel français.

4. UMR Paris Dauphine, Institut de Recherche pour le Développement (IRD).

5. I.e. les enquêtes sont menées depuis le pays d'installation des migrants : les États-Unis dans les cas des projets « New Immigrant Survey » et «Cuban and Mexican Immigrants in the US », menés par l'Université de Princeton.

6. I.e. les enquêtes sont menées depuis le pays d'origine des migrants, principalement en Amérique Latine ou dans les Caraïbes, comme dans le cas du «Project on Ethnicity ans Race in Latina America " (PERLA) ou du projet "Caribbean Urbanization in the Years of the Crisis » (CUIC), menés par l'Université de Princeton.

\section{INDEX}

Index géographique : Sénégal, France

Mots-clés : transnationalisme, relations avec le pays d'origine, transfert de fond, enquête

\section{AUTEUR}

\section{ILKA VARI-LAVOISIER}

Doctorante en Sociologie, École Normale Supérieure de Paris (France)

ilkav@princeton.edu 